\title{
Crispr, una herramienta para editar genomas
}

\author{
Crispr, a tool for edliting genomes
}

Brenda Gisela Martinez-Oliva ${ }^{1}$

\section{Resumen}

El artículo se centra en la utilización de la nueva herramienta, CRISPR (repeticiones palindrómicas cortas agrupadas a intervalos regulares), la cual permite editar los genomas de los seres vivos de manera más precisa que otras técnicas; a lo largo del artículo se mencionan trabajos relacionados con la detención de la angiogénesis, cáncer, Sarcoma de Kaposi en inmunodeficiencias, Parkinson, regeneración y modificación genética en humanos, todas estas investigaciones tiene en común la utilización de la herramienta CRISPR.

También se comenta las complicaciones éticas que conlleva utilizar esta tecnología en el ADN de células embrionarias humanas, que según diferentes criterios, podrían llevar a generar seres humanos "mejorados", es decir no solo sin susceptibilidad a enfermedades degenerativas o incurables, sino también modificados en aspectos físicos que no necesariamente estarían ligados a alguna patología.

Palabras clave: CRISPR, genoma, ADN, tecnología

\section{Abstract}

The article focuses on the use of the new tool, CRISPR (short palindromic repetitions grouped at regular intervals), which allows editing the genomes of living beings more accurately than other techniques; Throughout the article, works related to the arrest of angiogenesis, cancer, Kaposi's sarcoma in immunodeficiencies, Parkinson's, regeneration and genetic modification in humans are mentioned, all these investigations have in common the use of the CRISPR tool. You can also comment on the ethical complications that involve using this technology in the DNA of human embryonic cells, which according to different criteria, carry out improved human beings, that is not only without susceptibility to degenerative or incurable diseases, but also modified in physical aspects that are not linked to any pathology.

Keywords: CRISPR, genome, DNA, technology

$\mathrm{E}^{1}$ nombre CRISPR está resonando en varios escenarios científicos, pero ¿Qué es realmente y por qué está siendo estudiado y utilizado en la actualidad? En principio, el acrónimo CRISPR proviene del inglés clustered regularly interspaced short palindromic repeat que en español significa, repeticiones palindrómicas cortas agrupadas a intervalos regulares, entonces CRISPR es el nombre que recibe un locus (locus $=$ posición del gen $\mathrm{u}$ otra secuencia de ADN) del cromosoma bacteriano en donde se encuentran genes, con los cuales se ha creado una poderosa herramienta que permite manipular deliberadamente el $\mathrm{ADN}$ de cualquier organismo. Ahora el nombre CRISPR también identifica una técnica con la que se logra cambiar el genoma ${ }^{1}$. Por lo tanto, el nombre CRISPR se refiere a una sección de ADN en bacterias y arqueas, y también a la técnica que utiliza genes de esta sección como herramienta en la edición de genomas.

Estas secuencias repetidas que hoy se conocen como CRISPR fueron identificadas hace muchos años. Un grupo de científicos japoneses que estudiaba Escherichia coli ${ }^{2}$ y luego el español Francisco Mojica experimentando con Haloferax mediterranei (arquea), descubrieron estas secuencias ${ }^{3}$ CRISPR funciona como un sistema de defensa en estos

\footnotetext{
${ }^{1}$ Universidad Privada Abierta Latinoamericana https://orcid.org/0000-0002-5909-0245 ${ }^{*}$ Correspondencia a: Brenda Gisela Martinez-Oliva Correo electrónico: brendagisela000@gmail.com Recibido el 22 de enero de 2020. Aceptado el 25 de marzo de 2020.
}

microorganismos, equivalente a la inmunidad adquirida en animales ${ }^{1,4}$; un bacteriófago (virus que infecta bacterias) al infectar una célula le inserta ADN viral que se introduce en el ADN bacteriano, justamente en el sitio denominado CRISPR, por este mecanismo los ataques de los bacteriófago se graban con el tiempo, creando un archivo de infección, los trozos virales insertados son transferidos a la progenie de generación en generación para protegerlos contra los mismos virus que causaron la primera infección. La defensa de estos procariontes está mediada por genes del locus CRISPR, por lo que organismos tan sencillos se inmunizan contra reinfecciones por virus.

El locus CRISPR funciona como un registro de pedazos de ADN tomados del genoma de los bacteriófagos que previamente infectaron a la bacteria. Los genes asociados (Cas) son los que generan proteínas involucradas en la creación del archivo, Cas9 (CRISPR-associated 9) es la endonucleasa que destruye al ADN del bacteriófago reinfectante, la especificidad del sistema la proporciona el crARN (CRISPR ARN) que reconoce por apareamiento de bases a las secuencias nucleotídicas del ADN del fago, el tracrARN (trans-activating crRNA) sirve para ensamblar al híbrido crARN-ADN en el sitio activo de las Cas9 $9^{1}$. Por lo tanto la eficacia del sistema, es decir la forma activa de la endonucleasa Cas9 se presenta cuando existe un complejo entre los componentes: crARN, tracrARN y el ADN blanco (Figura 1).

La imagen superior muestra el complejo natural del Sistema CRISPR/Cas 9 sobre el ADN blanco. El crARN interactúa 


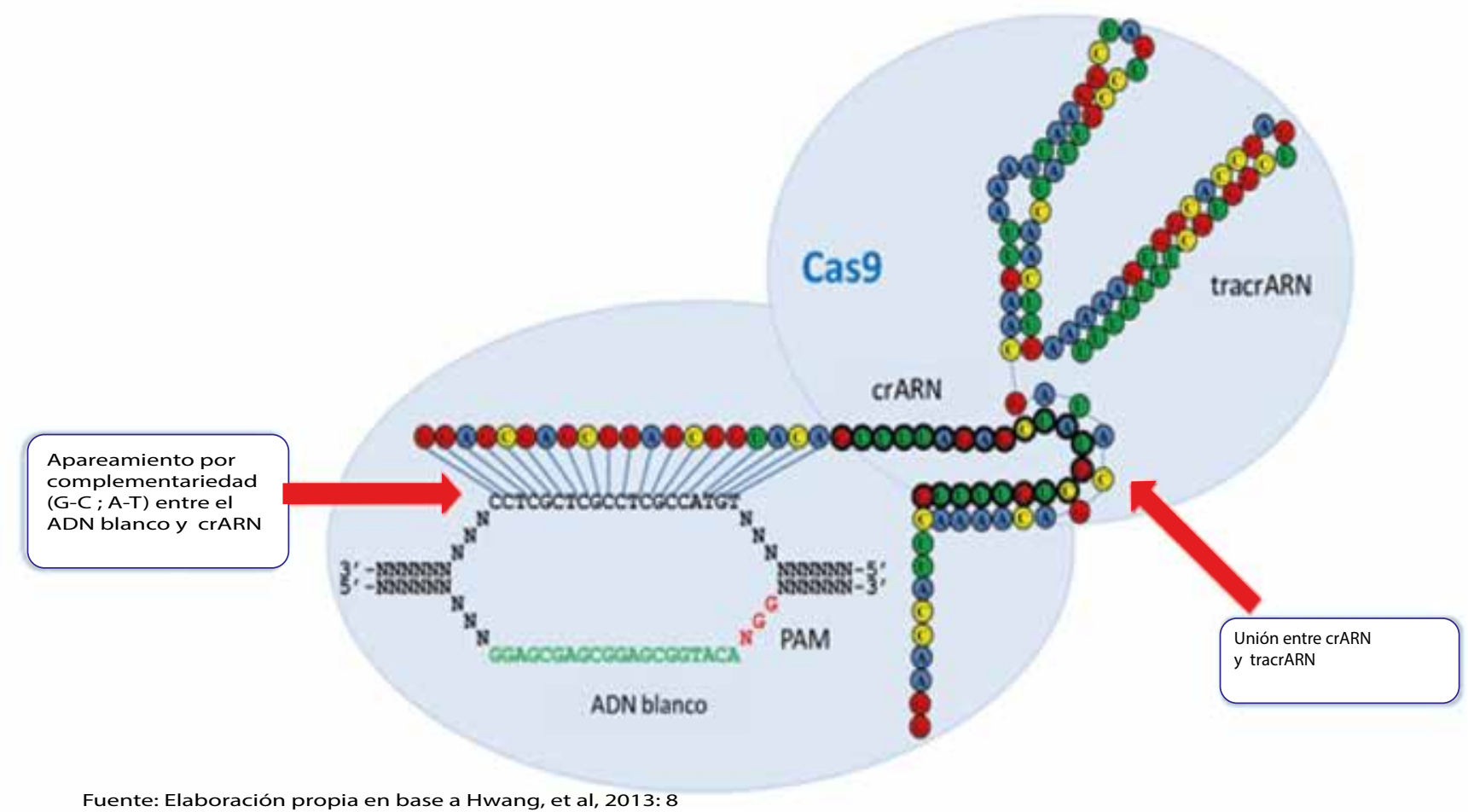

Figura 1. Representación esquemática de un complejo natural del sistema crispr/cas9 sobre un blanco de ADN

con la cadena complementaria del sitio blanco en el ADN (verde) el cual alberga una secuencia PAM (motivo adyacente protoespaciador (NGG de color rojo)), que es un componente de orientación esencial necesario para que la nucleasa corte el ADN invasor viral y no el ADN bacteriano. El plegamiento de las moléculas tracrARN y crARN mostradas en la imagen fueron predichas según el paquete de Software Mfold $20^{5} \mathrm{y}$ la asociación entre ambas (tracrARN y crARN) está basada en el modelo de Jinek ${ }^{6}$. Entonces, tracrARN es una cadena más larga de bases y proporcionan una estructura hairpin loop (tallo, bucle, horquilla), y sirve para ensamblar al híbrido crARN- ADN blanco en el sitio activo de la nucleasa Cas9; es importante notar que a pesar de que el complejo CRISPR está hibridado a una hebra de ADN, es capaz de cortar las dos hebras.

Es así que, Jennifer Doudna y Emmanuelle Charpentier (ganadoras del Premio Príncipe de Asturias 2015 aprovecharon estas características para diseñar la tecnología CRISPR/Cas 9 que está revolucionando la ingeniería genética. En la actualidad este sistema se utiliza para cortar zonas de interés del ADN e introducir secuencias deseadas que producirían genotipos o fenotipos deseados; ellas diseñaron un sgARN (single guide RNA o ARN guía) que presenta las mismas características que tracrARN y crARN, este sgARN dirige a la nucleasa hacia el ADN blanco con la misma precisión que lo harían crARN y tracrARN de una bacteria, para obtener un sgARN con especificidad contra el blanco genómico de elección, se debe diseñarlo con complementariedad de bases, es decir G-A y C-T (reglas de Watson y Crick), entonces al introducir el sgARN y la Cas9 al núcleo eucarionte donde se encuentre el ADN blanco, esté será cortado en ambas hebras del ADN generando un DSB (double stranded break o rotura de doble cadena), este tipo de cortes son reparados por el sistema NHEJ (nonhomologous end-joining o unión final no homóloga) que no es preciso, ocasionando que el ADN sufra inserción o deleciones de bases (indels) alrededor del DSB, que podrían en algunos casos producir pérdida de la función del ADN blanco ${ }^{1}$. La inactivación de un gen se utiliza para generar organismos knockout, en los cuales se estudia la función del gen bloqueado, también en el DSB se pueden insertar genes de interés para modificar alguna función o estructura en los organismos deseados y por ejemplo, producir algún producto biotecnológico de interés.

El sistema CRISPR/Cas 9 es una herramienta poderosa, fácil de utilizar y altamente específica para el genoma de eucariotas ${ }^{7}$ especialmente para células humanas ${ }^{8}$.

\section{Investigaciones actuales que utilizan el sistema CRISPR}

Entonces, en la actualidad existen diferentes investigaciones que están usando la herramienta CRISPR, a continuación se presentan algunos de los ejemplos más actuales y controversiales:

1.1. Virus adeno-asociado (AAV) - CRISPR/Cas9 para reducir la expresión de vegrfr 2 y bloquear la angiogénesis in vitro

La angiogénesis es un proceso natural que se refiere a la 
formación de vasos sanguíneos en tejidos del cuerpo, en algunos casos este proceso puede estimularse para curar heridas, o mejorar problemas cardíacos y su disminución o inhibición es utilizada como tratamiento en el cáncer y otras enfermedades.

La angiogénesis anormal es un componente de muchas enfermedades, incluyendo la degeneración macular, retinopatía diabética, artritis, crecimiento de tumores y metástasis. El propósito de la siguiente investigación realizada en Japón fue la de examinar si el sistema AAV-CRISPR/Cas9 podía reducir la expresión de VEGFR2 (receptor 2 del factor de crecimiento endotelial vascular) en el endotelio vascular de células humanas in vitro, los resultados mostraron que este sistema redujo la expresión de VEGFR 2 en un 80 \% y además bloqueó completamente la activación de VEGF (factor de crecimiento endotelial vascular) por Akt (un tipo de proteína serina - treonina cinasa, también conocida como proteína cinasa $\mathrm{B}$, participa en el metabolismo, proliferación celular, señalización celular y apoptosis), también redujo la proliferación, migración y formación de tubos de células endoteliales microvasculares de la retina humana ${ }^{9}$. Entonces, el sistema AAV - CRISPR/Cas 9 demostró ser una potencial herramienta para inhibir la angiogénesis patológica y así mejorar el estado de las enfermedades donde la formación de vasos sanguíneos es un factor fundamental de la patogénesis.

1.2 Aplicación y optimización de CRISPR-cas9 mediante ingeniería genética en ajolote (Ambystoma mexicanum)

El Ajolote es un tipo de salamandra que tiene una capacidad regenerativa, si pierde una extremidad, a las semanas se genera una nueva, incluso se regeneran tejidos complejos como los huesos, nervios, partes del cerebro y médula espinal, es un modelo animal para la comprensión de mecanismos de regeneración. Los científicos aseguran que la clave está en su genoma, sin embargo como el New York Times, citado por el periódico UNAM Global, señala, el ajolote cuenta con 32000 millones de pares de bases ${ }^{10}$, diez veces más que el genoma del ser humano (3 millones de pares de bases), por lo que estudiar su genoma es aún un reto.

Investigadores en China describieron un protocolo optimizado para crear ajolotes genéticamente modificados usando el sistema CRISPR/Cas9. Con este protocolo, se pueden obtener individuos que albergan una mutación de desplazamiento de marco de tipo homocigoto, lo que permite el análisis de fenotipos en esta generación ${ }^{11}$. Es decir, la manipulación genómica del ajolote es esencial para comprender el desarrollo y la fisiología de la regeneración, este protocolo puede aplicarse potencialmente a otros modelos animales, especialmente a organismos con un transcriptoma (ARNm transcrito en ciertas circunstancias, de forma global) bien determinado pero que carece de un genoma (conjunto de genes y disposición de los mismos en la célula) bien caracterizado.

1.3 CRISPR/Cas 9 en la terapia del cáncer: esperanzas y desafíos
El término cáncer es usado para describir a un conjunto de enfermedades en las que se observa un proceso desmedido y descontrolado en la división de células del cuerpo, el cáncer resulta de tres procesos: proliferación de un grupo de células (tumor o neoplasia), invasión de este grupo hacia los tejidos adyacentes y metástasis a tejidos y órganos más lejanos, además se produce por anormalidades en el material genético. La técnica CRISPR/Cas9 ha demostrado ser una herramienta potencial para descubrir nuevos blancos e interacciones genéticas y moleculares en la terapia del cáncer, proveyendo una visión acerca de cómo los tumores responden al tratamiento farmacológico, además esta herramienta puede ser usada para crear células inmunes y virus oncolíticos para inmunoterapias ${ }^{12}$.

El cáncer es la segunda causa de muerte a nivel mundial $\mathrm{y}$ aún se siguen estudiando los diferentes mecanismos involucrados en crecimiento celular anormal, el sistema CRISPR está siendo utilizado para estudiar los mecanismos desconocidos y crear posibles curas para este conjunto diverso de enfermedades con mecanismos moleculares similares en algunos aspectos y diferentes en otros.

Una investigación desarrollada en Australia utilizó CRISPR/ dCas9 (variante del CRISPR/Cas descrito, pero que funciona con el mismo mecanismo) en un panel de líneas celulares humanas de melanoma y cáncer de mama triple negativo (no expresa genes para el receptor de estrógeno, progesterona ni receptor 2 del factor de crecimiento epidérmico human HER2/neu); esta investigación plantea que la expresión de PTEN (fosfatidilinositol-3,4,5-trisfosfato 3-fosfatasa-enzima que actúa como supresor de cáncer gracias a su actividad lípido-fosfatasa) se pierde en diferentes tipos de cánceres, incluso pequeños cambios en la actividad de PTEN afectan la susceptibilidad y el pronóstico. Esto puede proporcionar un enfoque terapéutico alternativo para los cánceres agresivos que son refractarios a tratamientos actuales ${ }^{13}$. Es así que el estudio de las diferentes proteínas involucradas en la iniciación, promoción, transformación o progresión del cáncer puede brindar nuevas dianas terapéuticas y mejor comprensión de mecanismos para generar nuevas terapias.

\subsection{Reducción de la latencia del herpesvirus asociado a} Sarcoma de Kaposi

El herpes virus humano tipo 8 asociado al sarcoma de Kaposi (KSHV) es el agente etiológico del sarcoma de Kaposi (KS), el cual es un cáncer que genera tejido anormal en forma de parches que crecen debajo de la piel en diferentes zonas como la boca, nariz, garganta, etc. Este cáncer define el diagnóstico de SIDA en personas infectadas por el VIH-1 o en pacientes inmunosuprimidos con trasplantes; en la actualidad no existe tratamiento efectivo contra el KS, por lo tanto la tasa de supervivencia es baja; el KSHV establece una infección latente en el huésped y constituye un gran desafío para el tratamiento del KS, el Sistema CRISPR/Cas9 está siendo utilizado para el estudio de un antígeno nuclear asociado a la latencia (LANA), el cual es absolutamente necesario en el mantenimiento, replicación y segregación de los episomas (molécula de ADN 
capaz de replicarse de manera autónoma en el citoplasma de la célula hospedadora o cuando está integrada físicamente al cromosoma de esta) de KSHV durante la mitosis, lo que hace de LANA un objetivo ideal para la edición con CRIPSR/Cas9.

Un estudio en Nebraska - USA, fue el primero en demostrar la viabilidad de utilizar el sistema CRISPR/Cas9 dirigido a LANA de KSHV con un sistema de administración de adenovirus para interrumpir la latencia de KSHV en líneas celulares epiteliales y endoteliales infectadas, sentando la base para una estrategia viable en países donde los pacientes presentan lecturas altas de carga viral, también podría utilizarse esta estrategia contra otros virus tumorigénicos ${ }^{14}$. Es importante estudiar la capacidad de infección de los adenovirus y su asociación con CRISPR, por ejemplo, el efecto es menor cuando se trabaja con células B del sistema inmune debido a la baja eficacia de infección.

\subsection{CRISPR/Cas 9 como protector de la muerte neuronal en un modelo de la enfermedad de Parkinson}

El Parkinson es una enfermedad mortal con avance neurodegenerativo causada por factores genéticos y/o ambientales. Estos pacientes presentan un trastorno en el movimiento por la destrucción progresiva crónica del Sistema Nervioso en varias zonas del cerebro; las proteínas (cuerpos de Lewy) se depositan en el área de la sustancia negra (SN) produciendo apoptosis en las neuronas dopaminérgicas de esta zona, frente a esto, el cuerpo estriado (CS) no recibe ninguna señal de la SN para reaccionar ante el movimiento, si el problema continúa se produce una atrofia por desuso.

Se ha sugerido que las respuestas inflamatorias contribuyen a la enfermedad, una investigación realizada el 2003 en Estados Unidos, estudió las neuronas dopaminérgicas afectadas, indicando que estas acumulan neuromelanina (dopamina oxidada y polimerizada), las microglias (son el soporte de las neuronas y eliminan desechos celulares) por fagocitosis internalizan moléculas de neuromelanina, activándose y produciendo mediadores inflamatorios y moléculas tóxicas para las neuronas del rededor, la exposición prolongada de la microglia a la neuromelanina podría proporcionar un daño amplificador que acelera la enfermedad, además la investigación reveló que existe un receptor específico expresado por las microglias para este proceso, llamado RAGE (receptor transmembrana para productos finales de glicación avanzada), el cual puede ser usado como una diana terapéutica en el tratamiento del Parkinson, además este receptor también está involucrado en otras enfermedades como la Diabetes, Ateroesclerosis y Alzheimer ${ }^{15}$. En febrero del 2019, una investigación en Corea utilizó esta diana terapéutica, los investigadores generaron células madre mesenquimatosas derivadas de la sangre del cordón umbilical (UCB-MSC) que secretan sRAGE (receptor soluble) el cuál evita la unión de este mismo receptor con su ligando en la célula, la producción de este tipo de células se logró mediante el método de edición de genes, CRISPR/Cas 9. Estas células UCB-MSC fueron trasplantadas al Corpus Striatum en un modelo animal (ratones con Parkinson inducido por rotenona); los resultados indican que se redujo la muerte neuronal y el movimiento mejoró, por lo que este enfoque terapéutico basado en UCB -MSC podría ser una estrategia de tratamiento para el Parkinson ${ }^{16}$. Es así que gracias a la tecnología CRISPR son posibles estas investigaciones que permiten esbozar nuevos tratamientos para enfermedades neurodegenerativas.

\subsection{Modificación genética en humanos utilizando CRISPR}

El año 2018 el científico He Jiankui anunció al mundo el nacimiento de dos gemelas genéticamente modificadas utilizando tecnología CRISPR. El periódico SINC (la ciencia es noticia) en noviembre del 2018 publicó un artículo indicando que investigadores de la Universidad de Ciencia y Tecnología del Sur (SUSTech), en China, anunciaron haber creado dos niñas gemelas modificadas con la herramienta de corta y pega genético CRISPR, un día después del anuncio, la propia Universidad emitió una denuncia de mala praxis al director del estudio, He Jiankui; estos científicos eliminaron el gen CCR5 con el objetivo de crear resistencia al VIH, viruela y cólera ${ }^{17}$. El CCR5 es un correceptor que el VIH necesita para fusionar su membrana con la célula huésped (células dendríticas y linfocitos $\mathrm{T} \mathrm{CD} 4+$ ) e introducir su material genético (ARN), entonces, al eliminar el gen CCR5 no se transcribe la proteína CCR5 por lo tanto el VIH no ingresa a la célula y la persona sería inmune al virus. Sin embargo este tipo de manipulación podría conllevar efectos no deseados que podrían evidenciarse en los años siguientes.

El biólogo molecular Lluis Montoliu, del Centro Nacional de Biotecnología, declaró al periódico SINC que, esta modificación realizada por He Jiankui y su equipo no sirve para corregir una anomalía genética del embrión que podría producir una enfermedad grave y/o incurable, sino al contrario se trata de un experimento de mejora genética que pretende crear personas con capacidades distintas ${ }^{17}$; por lo tanto, en este caso se pretende crear una estirpe nueva de seres humanos que serán en teoría, inmunes al VIH; las implicaciones éticas en este experimento son varias, ya que en realidad no solo se alteró el genoma de las niñas sino también de su descendencia, y en realidad lo que se quiere es que las terapias desarrolladas sean eficaces y ante todo seguras. Según reporte del Departamento de Salud y Servicios Humanos de EE.UU, el científico He Jiankui indica que, "En esta búsqueda global cada vez más competitiva de aplicaciones para la edición de genes, esperamos destacar", sin embargo hasta la fecha no existe la publicación formal del experimento en alguna revista científica $^{18}$. Este experimento ha sido desarrollado ignorando las inquietudes de la comunidad científica internacional en relación a la manipulación de líneas germinales humanas.

\section{Discusión}

CRISPR nos posiciona un paso adelante en la temática de la edición genética y en los últimos 5 años está siendo intensamente aplicada en humanos, animales, vegetales, bacterias y virus, sin embargo estas investigaciones abren debates de tipo ético, social y económico por el posible 
impacto a nivel mundial.

Esta tecnología se está utilizando mayormente para corregir diversos defectos genéticos en personas adultas, por lo tanto los errores en el material genético no se trasmitirían a la siguiente generación; la utilización de esta tecnología es sencilla; con programas bioinformáticos y técnicas de biología molecular se ampliaría la utilización, además de lo expuesto se considera una técnica relativamente barata, por lo tanto podría ser la promesa para aquellas personas enfermas con problemas incurables y discapacitantes.

CRISPR se puede aplicar a varias patologías como las leucemias, problemas cardíacos, renales, hepáticos, cánceres, etc., sin embargo existe la posibilidad de que sea utilizado con fines eugenésicos o para diseñar seres humanos con capacidades diferentes.

$\mathrm{Si}$ se pueden curar enfermedades también podrían mejorarse condiciones como la vejez, mala visión, calvicie, entre otras. Como lo indica John Harris en su libro Superman y la Mujer Maravilla: las dimensiones éticas de la biotecnología humana, mencionado por Bergel "Nos hallamos al borde de una nueva revolución con un poder asombroso. La revolución de la biología molecular nos dará un alcance sin precedentes. Nos permitirá fabricar nuevas formas de vida bajo pedido, formas de vida de todo tipo. La decisión que se nos plantea no es la de usar o no este poder, sino cómo y hasta qué punto"19. En este sentido, existen diversas respuesta para responder el "cuando", "cómo" y "hasta qué punto", las cuales dependerían de cada país, ya que cada nación cuenta con sus propias leyes, reglamentos e ideologías que al final serían la base para determinar el alcance de estas investigaciones que pretenden "mejorar" la raza humana, con esto se anticipan problemas éticos.

Se podría pensar que la edición genética es más ética que la del diagnóstico preimplantacional ya que implica la reparación y no la destrucción del embrión, entonces surge a pregunta ¿Cuán ético es generar humanos perfectos? ${ }^{20}$, por otro lado, ¿Esta tecnología será accesible para todos y sin restricciones? ¿Se generará desigualdad social? ¿Existen leyes que rijan la manipulación genética en todos los países y estas son suficientes?

Los futuros profesionales deben ser formados con competencias claramente identificas y con compromiso ético-social que respondan a los problemas emergentes, bien lo dijo Johann Wolfgang von Goethe "Con el conocimiento se acrecientan las dudas".

\section{Referencias bibliográficas}

1. López, C. F. (2015). CRISPR, el sueño divino hecho realidad. Revista de la Facultad de medicina de la UNAM 58 (5): 55-60

2. Ishino, Y., Shinagawa, H., Makino, K., Amemura, M., y Nakata, A. (1987). Nucleotide sequence of the iap gene, responsible for alkaline phosphatase isozyme conversion in Escherichia coli, and identification of the gene product. Journal of Bacteriology, 169(12):5429-5433.

3. Mojica, F. J. M., Juez, G., y Rodríguez-Valera. (1993). Transcription at different salinities of Haloferax mediterranei sequences adjacent to partially modified PstI sites. Mol Microbiol 9(3):613-621

4. Koonin, E.V., y Makarova, K. S. (2013). CRISPRcas: evolution of an RNA-based adaptive immunity system in prokaryotes. RNA Biol 10(5):679-686.

5. Hwang, W. Y., Yanfang, F., D., R., Maeder, M., Tsai, S. Q., Sander, J. D., et al. (2013). Efficient in vivo Genome Editing Using RNA-Guided Nucleases. Nat Biotechnol, 31(3): 227-229

6. Jinek, M., Chylinski, K., Fonfara, I., Hauer, M., Doudna, J., y Charpentier, E. (2012). A programmable dual RNA-guided DNA endonuclease in adaptive bacterial immunity. Science, 337(6096): 816-821.

7. Doudna, J., y Charpentier, E. (2014). The new frontier of genome engineering with CRISPR-Cas 9. Science, 346(6213):346-1077

8. Sanjana, N., Shalem, O., y Zhang, F. (2014).
Improved vectors and genome-wide libraries for CRISPR screening. Nat Methods, 11(8): 783-784

9. Wu, W., Duan, Y., Ma, G., Zhou, G., ParkWindhol, C., D’Amore, P. A., et al. (2017). AAVCRISPR/Cas9-Mediated Depletion of VEGFR2 Block Angiogenesis In Vitro. Invest Ophthalmol Vis Sci 58(14): 6082-6090

10. UNAM Global (3 de Febrero de 2018). El ajolote tiene el mayor genoma del mundo y da una pista sobre sus cualidades regenerativas. Recuperado de http://www.unamglobal.unam. $\mathrm{mx} / \mathrm{p}=32588$ (Fecha de consulta 03/03/2019)

11. Fei, J. F., Lou, W. P., Knapp, D., Murawala, P., Gerber, T., Taniguchi, Y., Nowoshilow, S., Khattak, S., y Tanaka, E. M. (2018). Application and optimization of CRISPR-Cas9-mediated genome engineering in axolotl (Ambystoma mexicanum). Nature Protocols, 13:2908-2943.

12. Martinez-Lage, Puig-Serra, Menendez, Torres-Ruiz y Rodriguez-Perales, 2018. CRISPR/ Cas9 for Cancer Therapy: Hopes and Challenges. Biomedicines 6 (4): 105

13. Moisés, C., Nuget, F., Waryah, C., Garcia-Bloj, B., Harvey, A., y Blancafort, P. (2018). Activación de la expresión del supresor de tumores PTEN con el sistema CRIPSR/dCas9. Mol Ther Nucleic Acids 14: $28-300$

14. Tso, F. Y., West, J.T., y Wood, C. (2019). Reduction of Kaposi's sarcoma-associated herpesvirus latency using CRISPR-Cas9 to edit the latency-associated nuclear antigen gene. J virol

\section{3(7): e02183-18}

15. Fen, L. L. (2003). The Michel J. Fox Foundation for Parkinson's Research. Entendiendo el Parkinson. Recuperado de https://www. michaeljfox.org/foundation/researchers. php?id=463 (Fecha de consulta 06/05/2019)

16. Lee, J. B., Bayarsaikhan, D., Arivazhagan, R., Park, H., Lim, B., Gwak, P., Jeong, Goo-Bo, Lee, J., Byun K. y Lee B. (2019). CRISPR/Cas9 Edited sRAGE-MSCs Protect Neuronal Death in Parkinson's Disease Model. Int J Stem Cells 12(1) 114-124

17. SINC (26 de Noviembre de 2018). Un científico chino dice haber creado bebés modificados con CRISPR. Recuperado de https://www.agenciasinc. es/Noticias/Un-cientifico-chino-dice-habercreado-bebes-modificados-con-CRISPR (Fecha de consulta 06/05/2019)

18. Departamento de Salud y Servicios Humanos de EE.UU. (2019). CCR5. Recuperado de https:// infosida.nih.gov/understanding-hiv-aids/ glossary/979/ccr5 (Fecha de consulta 06/11/2019) 19. Bergel, A. D. (2017). El impacto ético de las nuevas tecnologías de edición genética. Rev. Bioét 25(3): 454-461

20. Becu, Damasia, (2017). El Sistema Crispr/Cas9 ¿Cambiará el genoma de la humanidad? Medicina (Buenos Aires) 77 (6): 521-523 